\title{
Analysis of Several High-Resolution Infrared Bands of Spiropentane, $\mathrm{C}_{5} \mathrm{H}_{8}$
}

\author{
A. Maki ${ }^{\mathrm{a}}$, J.E. Price ${ }^{\mathrm{b}}$, Jared Harzan $^{\mathrm{b}}$, J.W. Nibler ${ }^{\mathrm{b}, * *}$, \\ A. Weber ${ }^{\mathrm{c}}$, T. Masiello ${ }^{\mathrm{d}}$, and T.A. Blake \\ ${ }^{a} 1501224^{\text {th }}$ Ave., S.E, Mill Creek, WA, 98012 \\ ${ }^{b}$ Department of Chemistry, Oregon State University, Corvallis, OR, 97332-4003 \\ ${ }^{c}$ Sensor Science Division, National Institute of Standards and Technology, \\ Gaithersburg, MD 20899 \\ ${ }^{d}$ Department of Chemistry \& Biochemistry, 226 Science Building, Eastern Washington \\ University, Cheney, WA 99004 \\ ${ }^{e}$ Pacific Northwest National Laboratory, P.O. Box 999, Mail Stop K3-61, Richland, WA \\ 99352
}

Number of text pages: 18

Number of Tables: 5

Number of Figures: 4

** Corresponding author.

E-mail address: Niblerj@ chem.orst.edu

FAX: +1- 541-737-2062

Keywords: Spiropentane; High-resolution infrared spectrum; Rovibrational constants; DFT study; Anharmonic frequencies 


\begin{abstract}
The high-resolution infrared absorption spectrum of spiropentane $\left(\mathrm{C}_{5} \mathrm{H}_{8}\right)$ has been measured from 200 to $4000 \mathrm{~cm}^{-1}$, and a detailed analysis is presented for eight bands in the region from 700 to $2200 \mathrm{~cm}^{-1}$. Two fundamental perpendicular bands were analyzed, $v_{22}$ and $v_{24}$ near 1050 and $780 \mathrm{~cm}^{-1}$, respectively, along with two fundamental parallel bands, $v_{14}$ and $v_{16}$ near 1540 and $990 \mathrm{~cm}^{-1}$, respectively. Two other fundamentals, $v_{17}$ and $v_{23}$, are seen as intense overlapping bands near $880 \mathrm{~cm}^{-1}$ and are Coriolis-coupled, producing a complex mixture in which only $P$ branch transitions could be tentatively assigned for $v_{17}$. In addition, three binary combination bands were fit at about 1570,2082 , and $2098 \mathrm{~cm}^{-1}$ which are assigned as either $2 v_{24}$ or $v_{5}+v_{16}$ in the first case, $v_{4}+v_{22}$ in the second case, and $2 v_{22}$ in the latter case. The two $l$-type resonance constants, $q^{+}$and $q^{-}$, were determined for each of the two perpendicular fundamentals $v_{22}$ and $v_{24}$. Those two constants were also responsible for splittings observed in the $K=3$ levels of $v_{24}$. For the ground state the order of the split $K=2 B_{1} / B_{2}$ levels has been reversed from that reported previously, based on the measurements and assignments for the $v_{24}$ band. Rovibrational parameters deduced from the analyses are compared with those obtained from density functional Gaussian calculations at the anharmonic level.
\end{abstract}




\section{Introduction}

As the simplest bicyclic ring compound, the structure and physical properties of spiropentane are of special interest. The ring strain in spiropentane $\left(265 \mathrm{~kJ} \mathrm{~mol}^{-1}\right)$ is more than twice that of cyclopropane $(115 \mathrm{~kJ}$

$\mathrm{mol}^{-1}$ ), the excess being attributed to the spiro-arrangement about the central carbon.[1] Despite this strain, spiropentane is remarkably stable up to

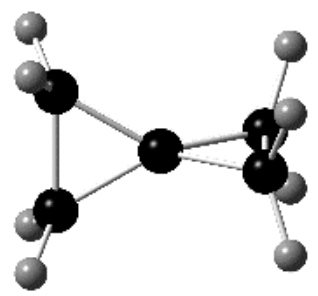
temperatures of about $350{ }^{\circ} \mathrm{C}$. Spiropentane is a symmetric top and it, along with allene and cyclobutane, are the only molecules of molecular symmetry $D_{2 \mathrm{~d}}$ known to us for which high resolution vibration-rotation spectra have been analyzed to obtain accurate molecular constants. Such information is particularly useful in assessing the merits of quantum calculations of the anharmonic potential function using programs such as Gaussian.

The infrared spectrum of spiropentane was first measured by Murray and Stevenson [2]. Subsequently, Cleveland et al. [3] and then Burns and McGavin [4] reported the results of infrared and Raman spectral measurements. Gaussian calculations were used by Schaad and Jarzecki [5] to verify the assignment of the fundamental vibrational wavenumbers and similar calculations were extended in a recent paper from this laboratory [6]. In that study of the highresolution infrared spectrum of spiropentane, partial analyses of several fundamental bands and two combination bands were used to give ground state combination-differences to determine accurate values for the rotational constants of spiropentane in the ground state. We have now extended those analyses and added the analysis of another fundamental band, a perpendicular band, $v_{22}$, near $1052 \mathrm{~cm}^{-1}$. Theoretical $v_{0}$ and intensity values for the fundamentals, along with mode numbering, symmetry, and assigned values [4] are given in our previous paper [6].

The spectrum of spiropentane is cluttered with hot band transitions due to the two low wavenumber fundamental bands, $v_{12}$ at about $300 \mathrm{~cm}^{-1}$ and $v_{25}$ at about $308 \mathrm{~cm}^{-1}$. At room temperature about $61 \%$ of the molecules are in the ground state while in sum about $14 \%$ are in the two lowest vibrational states, with the remaining $25 \%$ in other vibrational states. Consequently, the spectrum will generally have many unassigned transitions. The rovibrational analysis of the low $K$ levels for degenerate states in spiropentane is further complicated by splittings produced by two $l$-resonance constants $q^{+}$and $q^{-}$(only one such term, $q^{+}$, occurs for symmetric tops with three-fold symmetry). In our earlier paper [6] a very small splitting of the $K$ 
$=2$ levels was also observed for one parallel band, $v_{16}$, and used to determine the $\Delta_{2}$ splitting constant in the ground state. In the present work the larger $l$-resonance splitting of the $k l= \pm 1$ and $k l= \pm 3$ levels of two degenerate fundamentals, $v_{22}$ and $v_{24}$, was observed. From the analysis of these splittings it was necessary to revise the analysis of the splitting for the ground state and for $v_{16}$. These new measurements did not make a significant change to the absolute value of the $\Delta_{2}$ splitting constant for the ground state, but the order of the $B_{1}$ and $B_{2}$ levels has been changed.

\section{Experimental details}

The synthesis of our sample of spiropentane was described in the earlier paper [6]. The high-resolution infrared spectra were measured on the Bruker IFS 125HR Fourier transform spectrometer ${ }^{1}$ located at the Pacific Northwest National Laboratory. In recording the spectra a Globar light source, $\mathrm{KBr}$ beamsplitter, and either MCT or InSb detectors were used. The spectrometer and optical path outside of the absorption cell was evacuated. The sample was contained in a White-type absorption cell with a path length of $12.8 \mathrm{~m}$. Sample pressures between 150 and $16 \mathrm{~Pa}$ (1.1 and 0.12 torr) were used, depending on the intensity of the band being measured. Measurements were made from 200 to $4000 \mathrm{~cm}^{-1}$ with a resolution of about $0.002 \mathrm{~cm}^{-1}$. No transitions below $750 \mathrm{~cm}^{-1}$ or above $2100 \mathrm{~cm}^{-1}$ were fit, because no regular patterns were discerned among the bands that were strong enough to be measured.

The wavenumber scale for the spectra was based on $\mathrm{CO}_{2}[7]$ and $\mathrm{N}_{2} \mathrm{O}$ [8] gas reference samples measured immediately before or after the spiropentane spectrum was recorded. The calibration correction was typically less than $0.0002 \mathrm{~cm}^{-1}$ and it is believed that the absolute wavenumber accuracy is comparable.

As an example of the spectra obtained, Fig. 1 displays a high-resolution scan of the 780 $\mathrm{cm}^{-1}$ perpendicular band $v_{24}$. As shown at the top of the figure, the Gaussian calculations indicate that this mode is predominantly a rocking motion of the $\mathrm{CH}_{2}$ groups. The trace at the bottom shows the spectrum as calculated with the constants given in this paper.

\footnotetext{
${ }^{1}$ Certain commercial equipment, instruments, and materials are identified in this paper to adequately specify the experimental procedure. Such identification does not imply recommendations or endorsements by the National Institute of Standards and Technology or the Pacific Northwest National Laboratory, nor does it imply that the materials or equipment identified are necessarily the best available for the purpose.
} 


\section{Band Analyses}

\subsection{Rovibrational level expressions}

The analysis of the spectra used the same basic symmetric-top least-squares fitting program that was used for the earlier paper [6]. Since we have used no off-diagonal matrix elements that connect the $\mathrm{A}$ and $\mathrm{B}$ rotational levels, each matrix only includes rovibrational levels with either $A, B$, or $E$ symmetry. We give here all the basic equations that were used in the present work.

Neglecting interaction terms, the total rovibrational term value expression is given by

$$
E\left(\mathrm{v}_{\mathrm{m}}, \mathrm{v}_{\mathrm{n}}, \ldots, l_{\mathrm{m}}, l_{\mathrm{n}}, \ldots, k, J\right)=G\left(\mathrm{v}_{\mathrm{m}}, \mathrm{v}_{\mathrm{n}}, \ldots, l_{\mathrm{m}}, l_{\mathrm{n}}, \ldots\right)+F_{\mathrm{v}}\left(J, k, l_{\mathrm{m}}, l_{\mathrm{n}}, \ldots\right)
$$

All constants and terms are given in wavenumber units $\left(\mathrm{cm}^{-1}\right)$. For convenience we have chosen to base the vibrational term value expression, $G(\mathrm{v}, l)$, on the ground state so that $G(0,0)=G_{0}=0$

$\mathrm{cm}^{-1}$. If we then use $G_{0}$ for the zero point energy, the customary expression for the total vibrational term value becomes

$$
G_{0}+G(\mathrm{v}, l)=\sum \omega_{i}\left(\mathrm{v}_{i}+\frac{1}{2} d_{i}\right)+\sum_{i \leq j} x_{i j}\left(\mathrm{v}_{i}+\frac{1}{2} d_{i}\right)\left(\mathrm{v}_{j}+\frac{1}{2} d_{j}\right)+\sum_{t \leq t^{\prime}} x^{t t^{\prime}} l_{t} l_{t^{\prime}}+\ldots
$$

If there is no vibrational resonance to affect the band center, then the usual symbol that stands for the band center, $v_{0}$, is equivalent to $G(\mathrm{v}, l)^{\prime}-G(\mathrm{v}, l)^{\prime \prime}$.

The last term in Eq. (1) is the expression for the rotational term value. In its most general form, this standard energy (or term value) expression describing the rotational levels of a vibrational state of quantum number $\mathrm{v}$ is

$$
\begin{aligned}
F_{\mathrm{v}}\left(J, k, l_{i}\right) & =B_{\mathrm{v}} J(J+1)+\left(A_{\mathrm{v}}-B_{\mathrm{v}}\right) K^{2}-\sum k l_{i}\left[2 A \zeta_{i}-\eta_{i J} J(J+1)-\eta_{i k} K^{2}-\eta_{i J J} J^{2}(J+1)^{2}\right. \\
& \left.-\eta_{i J K} J(J+1) K^{2}-\eta_{i K K} K^{4}\right]_{\mathrm{v}}-D_{\mathrm{v} J} J^{2}(J+1)^{2}-D_{\mathrm{v} J K} J(J+1) K^{2}-D_{\mathrm{v} K} K^{4} \\
& +H_{\mathrm{v} J} J^{3}(J+1)^{3}+H_{\mathrm{v} J K} J^{2}(J+1)^{2} K^{2}+H_{\mathrm{v} K J} J(J+1) K^{4}+H_{\mathrm{v} K} K^{6}+\text { splitting terms. }
\end{aligned}
$$

In this paper, as is usual, lower case rotational quantum numbers are signed numbers while 
absolute values are used for upper case quantum numbers. Here, the rotational constants are given by

$$
B_{\mathrm{v}}=B_{e}-\sum_{i} \alpha_{i}^{B}\left(\mathrm{v}_{i}+1 / 2 d_{i}\right)+\ldots,
$$

with $-\alpha_{i}^{B}=B_{i}{ }^{\prime}-B_{i}{ }^{\prime \prime}=\Delta B$ and similar expressions describing the other rotational constants, $A_{\mathrm{v}}$, $D_{\mathrm{v} J}$, etc. The $D, H$ and $\eta$ terms account for centrifugal distortion effects. For the ground vibrational state and for all other vibrational states with $l=0$ treated in this paper, the splitting term is given by

$$
\text { splitting }= \pm \delta_{2 K} \Delta_{2 v}[J(J+1)][J(J+1)-2]
$$

which only affects the $K=2$ rotational levels. The first factor on the right hand side of Eq. (5) is the Kronecker delta symbol, $\delta_{2 K}$, which is zero if $K \neq 2$; the second symbol, $\Delta_{2 v}$, is the splitting constant which is very small, on the order of $10^{-9} \mathrm{~cm}^{-1}$ or less. Its determination requires the observation of transitions with high $J$ values, which is seldom the case. The only states that required such a splitting term were the ground state and $v_{16}$. For all other states the measurements did not extend to high enough values of $J$ to enable a determination of that splitting constant.

Since spiropentane has $D_{2 d}$ symmetry, for vibrational states with $l=1$, both the $k l=+1$ and the $k l=-1$ levels are split by $l$-type resonance constants $q^{+}$and $q^{-}$whose determination requires the use of the off-diagonal matrix elements

$$
\begin{aligned}
W_{2,2}=\left\langle\mathrm{v}_{\mathrm{t}}, J, k, l_{\mathrm{t}}|H| \mathrm{v}_{\mathrm{t}}, J, k \pm 2, l_{\mathrm{t}} \pm 2\right\rangle \\
=1 / 4\left[q_{t}^{+}+q_{t J}^{+} J(J+1)+q_{t K}^{+}\left(k^{2}+(k \pm 2)^{2}\right)\right]\left[\left(\mathrm{v}_{\mathrm{t}}+1\right)^{2}-\left(l_{t} \pm 1\right)^{2}\right]^{1 / 2} \\
\quad \times[J(J+1)-k(k \pm 1)]^{1 / 2}[J(J+1)-(k \pm 1)(k \pm 2)]^{1 / 2}
\end{aligned}
$$

and

$$
\begin{aligned}
W_{2,-2}= & \left.<\mathrm{v}_{\mathrm{t}}, J, k, l_{t} \pm 2|H| \mathrm{v}_{\mathrm{t}}, J, k \pm 2, l_{\mathrm{t}}\right\rangle \\
=1 / 4\left[q_{t}^{-}+q_{t J}{ }^{-} J(J+1)+q_{t K}{ }^{-}\left(k^{2}+(k \pm 2)^{2}\right)\right]\left[\left(\mathrm{v}_{\mathrm{t}}+1\right)^{2}-\left(l_{t} \pm 1\right)^{2}\right]^{1 / 2} & \\
& \times[J(J+1)-k(k \pm 1)]^{1 / 2}[J(J+1)-(k \pm 1)(k \pm 2)]^{1 / 2} .
\end{aligned}
$$


Those matrix elements also result in a splitting of the $k l=+3$ and $k l=-3$ levels, although this is much smaller than that for the $k l=+1$ and $k l=-1$ levels.

One degenerate state, $v_{24}$, required an additional matrix element, $W_{4,0}$, to improve the fit of the $K=1$ levels beyond the splitting provided by the above terms.

$$
\begin{aligned}
W_{4,0}=\langle v, J, k, l|H| v, J, k \pm 4, l\rangle=w_{4,0}[ & {[(J+1)-k(k \pm 1)]^{1 / 2}[J(J+1)-(k \pm 1)(k \pm 2)]^{1 / 2} } \\
& \times[J(J+1)-(k \pm 2)(k \pm 3)]^{1 / 2}[J(J+1)-(k \pm 3)(k \pm 4)]^{1 / 2},
\end{aligned}
$$

where $w_{4,0}$ is an off-diagonal splitting constant.

The sign of the $q^{+}$splitting constant follows the convention discussed by Cartwright and Mills [9] and used by others in the analysis of the spectrum of allene [10,11] and by us in previous work on $D_{3 h}$ symmetric top molecules such as propellane [12,13]. This convention was chosen by Cartwright and Mills to achieve agreement between experimental and theoretical signs for $q^{+}$for symmetric tops of $C_{3 v}$ symmetry and, as applied to molecules of $D_{2 d}$ symmetry, with $q^{+}$defined by our Eq. 6 , is that $q^{+}$is positive if the $B_{1}$ rovibrational level is above the $B_{2}$ level for even $J$ values, and the reverse for odd $J$ values. ${ }^{2}$ These authors explicitly avoid a choice of convention for $q^{-}$, but an operational choice was made for allene in Reference 11 in which $q^{-}$ is positive for even $J$ values if $A_{1}>A_{2}$. We have adopted this choice here. If sufficiently separated, transitions to both the $A_{1} / A_{2}$ levels are visible and can be identified because the intensity ratio should be that of the nuclear spin weights, 76/60. Similarly, transitions to $B_{1} / B_{2}$ split levels will have a 76/60 intensity ratio. More details regarding the ordering and signs of the $A_{1} / A_{2}$ and $B_{1} / B_{2}$ pairs are given in the discussion of each band.

\subsection{Ground state rotational constants}

In our earlier paper on spiropentane [6] the ground state rotational constants were given based on the combination-differences then available. The present work has added more than 1500 ground state combination-differences so a new fit has been made that includes all the

\footnotetext{
${ }^{2}$ The $\mathrm{W}_{2,2}$ matrix element in reference [9] has a leading negative sign whereas our Eq. (6) does not, nor does the $\mathrm{W}_{2,2}$ element defined in references [10,11]. To compensate for this, the level ordering convention given above is the opposite of that given in reference [9]; thus in both cases $q^{+}$has the same sign and can be compared to the theoretical expression given in [9].
} 
currently available data. Despite this increase in the data set size, the sextic centrifugal distortion $H$ constants remained poorly determined and hence were fixed at zero values. The results are given in Table 1 and these ground state constants were used in all the fits in the present work. The new ground state constants are only slightly different from the earlier values but are expected to be more accurate. The main difference is the splitting constant $\Delta_{2}$, for which a more accurate value was determined by the current analysis of the $v_{24}$ band and for which the positive sign agrees with the theoretical value if one uses the convention adopted for allene in Reference 11. A summary of our convention for order of levels for positive splitting constants is thus

$\begin{array}{llll}J \text { even } & \frac{q^{+}>0}{B_{1}>B_{2}} & \frac{q^{-}>0}{A_{1}>A_{2}} & \underline{\Delta_{2}}>0 \\ J \text { odd } & B_{1}<B_{2} & A_{1}<A_{2} & B_{1}>B_{2} .\end{array}$

\subsection{The $v_{24} 780 \mathrm{~cm}^{-1}$ perpendicular band}

The lowest wavenumber band studied in this paper is the $v_{24}$ perpendicular band centered at $779.126 \mathrm{~cm}^{-1}$. No other vibrational energy level is within $90 \mathrm{~cm}^{-1}$ of $v_{24}$ and so it is found, as expected, to be free of any perturbations. As seen in Figure 1, the spectrum is very crowded but the analysis was nonetheless straight-forward; Table 2 gives the constants found from a fit of over $5700 v_{24}$ transitions. Of special interest were the three important splitting constants, $q^{+}$and $q^{-}$for $v_{24}$ and $\Delta_{2}$ for the ground state. From the fit of the transition wavenumbers alone it is not possible to determine the signs of any of these splitting parameters, but these can be obtained with the sign conventions described above, in combination with intensity considerations discussed below. Included in the fit were all the constants given in Eqs. (6) and (7) as well as the small $w_{4,0}$ constant given in Eq. (8). The $w_{4,0}$ constant is similar to $\Delta_{2}$ for the ground state (both involve the $\mathrm{J}_{+}{ }^{4}+\mathrm{J}_{-}{ }^{4}$ operator), but for a degenerate state $w_{4,0}$ has its greatest effect on the $K=1$ levels. Once the signs of both $q^{+}$and $q^{-}$are known then the sign of $w_{4,0}$ is given by the fit.

The $B_{1} / B_{2}$ splitting for the $k l=+1$ levels of $v_{24}$ is caused by the $q^{+}$parameter via the $\mathrm{W}_{2,2}$ off-diagonal matrix element defined by Eq. 6. The observed transitions to those levels all originate from the $K=0$ levels of the ground state. Therefore, the correct order of the upper state $B_{1} / B_{2}$ levels is determined by the fact that the lower state $K=0$ levels are $A_{1}$ for even $J$-values and $A_{2}$ for odd $J$-values and the only allowed infrared rovibrational transitions are $B_{1} \leftrightarrow A_{1}$ or $B_{2} \leftrightarrow A_{2}$ or $E \leftrightarrow E$. The analysis shows that the $\Delta J=0 Q$-transitions all go to the lower of the two 
levels for $k l=+1$ while the $\Delta J= \pm 1 P$ - and $R$-transitions all go to the upper level. For this reason, without resorting to any intensity measurements, we have given $q^{+}$a negative sign to be consistent with the sign convention above.

Since the $k l=+1$ levels are coupled to the $k l=-3$ levels through the $q^{-}$constant, the latter levels should show a significant splitting with a $B_{1} / B_{2}$ order the same as for the $k l=+1$ levels, regardless of the sign of $q^{-}$. That expectation is confirmed by the calculated values which agree with the observed transitions. Similarly the $q^{+}$constant couples the $k l=-1$ levels to the $k l$ $=+3$ levels, so that the ordering of the $A_{1}, A_{2}$ levels is the same for $k l=-1$ and $k l=+3$ of $v_{24}$. (The $w_{4,0}$ constant is so small that it does not reverse that ordering for any of these cases).

From the splitting of the $k l=-1$ or +3 levels of $v_{24}$ we can determine very accurate values for the magnitude of $q^{-}$but not its sign. Only by observing the relative intensities of the split transitions can we determine the ordering of the $A_{1} / A_{2}$ levels and therefore the sign of $q^{-}$. It is important to be able to compare the intensity of two lines that are adjacent so that the effects of noise or overlapping with other lines are minimized. The splittings of the ${ }^{r} Q_{2}$ transitions for $J$ $>28$ are convenient for this since, though small, the resultant doublets can be resolved, see Figure 2. As an example, consider the doublet for $J=32$. Since we have accurate absolute values for $q^{+}$ and $q^{-}$we can calculate that, for instance, the splitting of the $k l=+3$ levels for $J=32$ is 0.0014 $\mathrm{cm}^{-1}$. We also have accurate values for $\Delta_{2}$ but not its sign. The calculated splitting of the $J=32$, $K=2$ levels for the ground state is $0.0026 \mathrm{~cm}^{-1}$. The observed splitting of the ${ }^{r} Q_{2}(32)$ transition is $0.0038 \mathrm{~cm}^{-1}$ which is approximately the sum of the upper and lower state splittings. As can be seen for ${ }^{r} Q_{2}(32)$ in Fig. 2, the more intense doublet member, which must be the $A_{1} \leftarrow B_{1}$ transition, must begin at the lower of the ground state split levels and go to the upper of the $v_{24}$ levels. Since the $B_{1} / B_{2}$ levels must be reversed if $J$ is raised or lowered by one unit, then the splitting of the corresponding ${ }^{r} R_{2}(31)$ and ${ }^{r} P_{2}(33)$ transitions should be given by the difference rather than the sum of the lower and upper state splittings, making the splitting quite small. This is consistent with the fact that we do not discern any indication of splitting of the ${ }^{r} R_{2}(31)$ and ${ }^{r} P_{2}(33)$ transitions.

A second example is provided by the odd $-{ }^{r} Q_{2}(41)$ transitions (not shown), which seem to be free of overlapping and have a separation of $0.01346 \mathrm{~cm}^{-1}$, which is equal to the sum of the calculated upper state splitting, $0.00647 \mathrm{~cm}^{-1}$, and the lower state splitting, $0.00694 \mathrm{~cm}^{-1}$. As for the odd- $J{ }^{r} Q_{2}(29)$ shown in Fig. 2, the lower component is stronger than the upper component, 
the reverse of the order for even- $J$ transitions. The corresponding splitting of the ${ }^{r} R_{2}$ and ${ }^{r} P_{2}$ transitions is again small and is only observed at much higher $J$-values.

The splittings of the ${ }^{p} P_{2},{ }^{p} R_{2}$, and ${ }^{p} Q_{2}$ transitions to $k l=-1$ levels are much larger and, although the lines are too widely-spaced and too badly overlapped to enable us to make good comparisons of the relative intensities, the splitting values are consistent with level orders deduced above. For example, the upper state splitting for $J=32$ is calculated to be $0.5739 \mathrm{~cm}^{-1}$ so that the observed splitting of the ${ }^{p} Q_{2}(32)$ transitions should be $0.5764 \mathrm{~cm}^{-1}$; the observed splitting is $0.5772 \mathrm{~cm}^{-1}$. For the ${ }^{p} R_{2}(31)$ transitions the measured splitting should be slightly smaller than 0.5739 and is observed to be $0.5721 \mathrm{~cm}^{-1}$. For ${ }^{p} P_{2}(33)$ the observed splitting is also smaller, $0.5732 \mathrm{~cm}^{-1}$. Similar comparisons can be made for higher $J$-values so that we are confident of the assignments and conclusions.

The result of the above observations is the conclusion that for the same $J$-values the ordering of the $B_{1} / B_{2}$ levels for $K=2$ in the ground state must be opposite to the ordering of the $A_{1} / A_{2}$ levels for $k l=-1$ of $v_{24}$. Therefore $\Delta_{2}$ should be positive and $q^{-}$should also be positive. Figure 3 summarizes the relative ordering of the energy levels for the ground state and for $v_{24}$. Although the figure is not to scale, the figure shows the correct relative ordering of the $A_{1} / A_{2}$ and $B_{1} / B_{2}$ split levels. Only a few $\Delta J=0$ transitions are shown in the figure but, of course, $\Delta J= \pm 1$ transitions were also observed.

\subsection{The $v_{22} 1051 \mathrm{~cm}^{-1}$ perpendicular band}

Another fundamental band that appears to be relatively free of perturbations is $v_{22}$ centered at $1051.36 \mathrm{~cm}^{-1}$. There are, however, many unassigned lines in this region and the density of lines is such that almost all lines are blended with other lines. It is predicted that $v_{4}$, and possibly $v_{3}$, should be close to $v_{22}$ but the spectrum does not show any obvious effects due to $v_{4}$ or any other nearby level.

As was the case for $v_{24}$ the $B_{1} / B_{2}$ splitting of the $k l=+1$ levels is rather large. The fact that the ${ }^{r} Q_{0}$ transitions all go to the upper $k l=+1$ level while the ${ }^{r} P_{0}$ and ${ }^{r} R_{0}$ transitions go to the lower $k l=+1$ levels means that the splitting constant, $q^{+}$, must be positive. That is opposite to what was found for $v_{24}$. This goes along with, but is probably not related to, the reversal of the sign of $A \zeta$.

The $A_{1} / A_{2}$ splitting of the $k l=-1$ levels is quite small and the overlapping with other 
transitions is so severe that it is not possible to determine the sign of $q^{-}$from intensity measurements. However, since we know the order of the ground state $K=2$ levels, the order of the $k l=-1$ levels of $v_{22}$ is determinable from measured transitions with $J>28$. The ${ }^{p} Q_{2}$ transitions are too badly overlapped to allow a good determination of the splitting, but that is not the case for a few ${ }^{p} P_{2}$ and ${ }^{p} R_{2}$ transitions. Those transitions all show that the splitting of the transitions is smaller than the splitting of the upper state, hence for a given $J$ the order of the $A_{1} / A_{2}$ levels for $k l=-1$ of $v_{22}$ must be opposite to the order of the $B_{1} / B_{2}$ levels of the ground state. Therefore the sign of $q^{-}$must be opposite to the sign of $\Delta_{2}$ for the ground state and is thus negative.

Because of the small value for the $q^{-}$constant, the splitting calculated for the $K=3$ levels is too small to observe. Instead, the splitting of the ${ }^{r} Q_{2},{ }^{r} P_{2}$, and ${ }^{r} R_{2}$ transitions must be primarily due to the splitting of the ground state $K=2$ levels. The calculated splitting of those levels is on the order of $0.0092 \mathrm{~cm}^{-1}$, compared with the observed splitting of $0.0094 \mathrm{~cm}^{-1}$ at the highest $J$ values observed, $J^{\prime \prime}=44$. In any case, those transitions are so badly overlapped with other transitions that intensity measurements cannot be used to independently verify the sign of $q^{-}$or the sign of the ground state $\Delta_{2}$ constant. The constants are given in Table 2 .

\subsection{The revised analysis of the $v_{16}$ parallel band near $993 \mathrm{~cm}^{-1}$}

Since the analysis of the band near $780 \mathrm{~cm}^{-1}$ reverses the order of the split $K=2$ levels for the ground state, we have reanalyzed the $993 \mathrm{~cm}^{-1}$ band. In the $P$-branch for the $K=2$ transitions the splitting is much smaller than that due to the lower state alone. In addition, the transitions that are displaced to lower wavenumbers between $J^{\prime \prime}=50$ and $J^{\prime \prime}=56$ are stronger for odd $J^{\prime \prime}$ values and weaker for even $J^{\prime \prime}$ values, in agreement with the lower state ordering shown in Figure 3. The transitions involving higher $J$-values are less reliable since they are weaker.

It is significant that the splitting of the ${ }^{q} P_{2}$ transitions is small, much less than the sum of the upper and lower state splittings that is predicted if the signs are identical for both states. The intensity pattern thus requires the upper state to have a splitting constant that is opposite in sign and smaller than that of the ground state. This sign reversal is not surprising because in going from the $A_{1}$ ground state to the $B_{2} v_{16}$ state the product of the vibrational symmetry species and the rotational symmetry for the $K=2$ rotational levels will reverse the order $B_{1}$ above (or below) $B_{2}$ to $A_{2}$ above (or below) $A_{1}$, i.e. $B_{2} \times B_{1}=A_{2}$ and $B_{2} \times B_{2}=A_{1}$. Therefore one would expect that 
for $K=2$ of $v_{16}$, the $A_{1}$ levels would be above the $A_{2}$ levels for even $J$. The important result is that there is no significant difference from the earlier analysis, except for the sign of $\Delta_{2}^{\prime}$, as shown by the constants given in Table 3 .

\subsection{The two parallel bands at 1539.5 and $1567.5 \mathrm{~cm}^{-1}$}

Two parallel bands are observed in the $1550 \mathrm{~cm}^{-1}$ region and, as seen in Figure 4, these have nearly the same intensity. The transitions involving the $K=0,1$, and 2 levels were not resolved for either band and both have many unexplained extra lines that may or may not be due to hot-band transitions. Only one fundamental band is expected, namely $v_{14}$, and the Gaussian calculations favor assignment of the lower band as $v_{14}$. The $K$-structure of the $P$ - and $R$-branch features is condensed and only 450 transitions could be assigned, giving, however, a very good rms deviation value of $0.00040 \mathrm{~cm}^{-1}$.

The assignment of the upper band is less clear and could be $v_{5}+v_{16}\left(B_{2}\right)$ or $2 v_{24}\left(A_{1}, B_{1}, B_{2}\right)$. A spectrum calculated in the first case (shown in Fig. 4) gives a good fit, although some band heading is seen in the $R$-branch at high $J$. If the upper state is $2 v_{24}$, it would have to be the $B_{2}$ component with either $l=+2$ or $l=-2$ and it would be coupled to the other $l$ components through $l$-type doubling matrix elements, as well as being coupled to $v_{14}$ through a vibrational resonance. In the fit of the upper band with this $2 v_{24}$ assignment, the inclusion of a $\Delta k= \pm 2, \Delta l=$ \pm 2 matrix element equivalent to a $q^{+}$term was used to couple the $l=0$ and $l= \pm 2$ levels. In addition, one should use a term equivalent to a $q^{-}$term to couple the $B_{1}$ and $B_{2}$ vibrational states, but that was not done because the data did not justify it. Despite this, the upper band fit was comparable to that obtained for the $v_{5}+v_{16}$ assignment and both alternatives are included in Table 4. The simulated spectrum for $2 v_{24}$ is not shown in Fig. 4, since it closely resembles that for shown for $v_{5}+v_{16}$. The rms deviations are essentially identical for both assignments so experiment does not allow a choice of one over the other.

Theory offers some guidance here, as shown in Table 5. For $v_{14}$, the theoretical $\triangle A$ and $\Delta B$ values are in good accord with experiment and the $v_{0}$ value is also reasonable, since a Fermi resonance between the $B_{2} v_{14}, 2 v_{24}$ and $v_{5}+v_{16}$ levels is expected, even though the wavenumber fits did not show evidence of such resonances. From the Gaussian calculations of level separations and the $\varphi_{14,24,24}$ and $\varphi_{5,14,16}$ cubic coupling terms, 39 and $11 \mathrm{~cm}^{-1}$, respectively, the resonance of $v_{14}$ with $2 v_{24}$ should be much more important than that with $v_{5}+v_{16}$ and this would 
compensate for the lower theoretical anharmonic intensity listed in table for $2 v_{24}$ compared to $v_{5}+v_{16}$. The $\Delta A$ and $\Delta B$ values of the upper $1567.5 \mathrm{~cm}^{-1}$ band are about twice that of the lower $1539.5 \mathrm{~cm}^{-1}$ band. The theoretical and experimental $\Delta A$ and $\Delta B$ values for the upper state are in poorer agreement for the $v_{5}+v_{16}$ assignment and the very large $\Delta H$ values found for this assignment also favors the $2 v_{24}$ assignment. Finally, though not analyzed, there is a weak $Q$ branch of a parallel band at about $1582.5 \mathrm{~cm}^{-1}$ and this is very close to the $v_{5}+v_{16}$ value of 1585 $\mathrm{cm}^{-1}$ that is predicted from theory. For these reasons, we favor the assignment of the 1567.5 $\mathrm{cm}^{-1}$ band as $2 v_{24}$, but we caution that the constants given in Table 4 for this state should be regarded as fitting parameters for an incomplete model.

\subsection{The two parallel bands near 2098 and $2145 \mathrm{~cm}^{-1}$}

Two parallel bands are observed in this region and the theoretical calculations predict only three $B_{2}$ states as possible upper state assignments in the range 2050 to $2200 \mathrm{~cm}^{-1}: v_{5}+v_{14}$, $2 v_{22}$, and $v_{7}+v_{11}$. The predicted parameters for each are shown in Table 5 and comparison of predicted intensities and the $\Delta A$ and $\Delta B$ values clearly favor the assignment of the more intense band centered at $2098.21 \mathrm{~cm}^{-1}$ as the $B_{2}$ component of $2 v_{22}$.

The analysis and assignment of the higher $2145 \mathrm{~cm}^{-1}$ band is less certain. This band is very weak and the high- $K$ features cannot be distinguished from the noise and clutter of hot band transitions. There is much structure in this region that is consistent with one or more perpendicular bands, but the analysis of this was not possible. However, a long series of $P$ branch-like parallel-band features extends from 2134 to $2142 \mathrm{~cm}^{-1}$ and a complementary series of $R$-branch-like features extends from 2149 to $2153 \mathrm{~cm}^{-1}$. The low- $J^{\prime}$ features for each $P$ and $R$ branch shade to higher wavenumbers as $K$ increases while the high- $J$ ' features shade to lower wavenumbers, with the $P$ - and $R$-branch features clearly sharpest for the $J^{\prime}=22$ transitions in both branches. This $J^{\prime}$-assignment is consistent with the weak $Q$-branch feature whose band center appears to be at $2145.747 \pm 0.010 \mathrm{~cm}^{-1}$. The change in shading of the features suggests that there is a crossing point at $J^{\prime}=22$ for two interacting bands. We note that the structure of the band is quite similar to that of the $v_{17}$ band we have previously analyzed for bicyclopentane [15]. In that case the shape of the bicyclopentane band was found to be due to the $x-y$ Coriolis interaction with the nearby degenerate fundamental $v_{11}$.

A similar Coriolis interaction is expected between $v_{7}\left(A_{2}\right)$ and $v_{21}(E)$ of spiropentane, 
which are calculated to be only a few $\mathrm{cm}^{-1}$ apart. Because of that, we expect that the combination states $v_{7}+v_{11}$ and $v_{11}+v_{21}$ will also be coupled through a $\zeta_{7,21}$ Coriolis term, which will result in a band that looks very much like Fig. 3 in Reference 15 for bicyclopentane. For spiropentane, the theoretical value of $\zeta_{7,21}$ is 0.4 , a value large enough that this the coupling term will be significant. As seen in Table 5, the $v_{0}$ and intensities are consistent with assignment of the band as $v_{7}+v_{11}$ but the experimental value deduced for $\Delta B$ is negative $\left(-0.90 \times 10^{-3} \mathrm{~cm}^{-1}\right)$, versus a positive value $\left(+0.06 \times 10^{-3} \mathrm{~cm}^{-1}\right)$ from theory. Also, the shading of the $Q$-branch to lower wavenumbers would seem to require a negative value for $\Delta A$, whereas the theoretical value is predicted to be positive.

The alternative assignment of the band is as $v_{5}+v_{14}\left(B_{2}\right)$ where a weak Coriolis interaction could also occur, with either $v_{11}+v_{21}$ or a $v_{16}+v_{21}(E)$ state at slightly higher energy. It may be significant that if the band is simply fitted as $v_{5}+v_{14}$, without any coupling, the sign disagreements for $\Delta A$ and $\Delta B$ disappear. The intensity predicted for this assignment is also slightly higher than for the $v_{7}+v_{11}$ choice. The predicted $v_{0}$ value is $26 \mathrm{~cm}^{-1}$ lower than the observed band center however, but such a lowering could be a consequence of Fermi mixing of the two $B_{2}$ states. All in all, the fits are judged to slightly favor the $v_{5}+v_{14}$ assignment but this conclusion should be regarded as tentative. ${ }^{3}$

\subsection{The perpendicular band near $2082 \mathrm{~cm}^{-1}$}

Buried in the $P$-branch region of the $2098 \mathrm{~cm}^{-1}$ parallel band is a series of $Q$-branch-like lines that seem to belong to a perpendicular band centered near $2083 \mathrm{~cm}^{-1}$. Matching those $Q^{-}$ branches is a series of strong $P$-branch lines that have an effective $A \zeta$ value close to the value found for $v_{22}$. Consequently we believe those transitions must belong to the binary band $v_{4}+v_{22}$ (E) which is predicted at $2092 \mathrm{~cm}^{-1}$. Since the Gaussian calculations indicate that $v_{3}$ is close to $v_{4}$, an alternative assignment is that this band is $v_{3}+v_{22}$, predicted at $2095 \mathrm{~cm}^{-1}$. However, as seen in Table 5, comparison of the predicted intensities, $\Delta A, \Delta B$ and $A \zeta$ values definitely favors the

\footnotetext{
${ }^{3}$ The experimental wavenumber values assigned for spiropentane by Burns and McGavin [4] suggest other combinations in this region involving $v_{3}, v_{8}$, and $v_{11}$ fundamentals. These are not considered here since, as discussed in our earlier paper [6], we believe these to be misassignments since their assigned values differ significantly from the theoretical values, by $-119,-85$, and $+116 \mathrm{~cm}^{-1}$, respectively.
} 
$v_{4}+v_{22}$ assignment. Assuming that assignment is correct, it implies that $v_{4}$ is at about $1030 \mathrm{~cm}^{-1}$, close to the $1037 \mathrm{~cm}^{-1}$ Raman feature assigned as $v_{4}$ for liquid spiropentane [3].

Since the $v_{3}+v_{22}$ and $v_{4}+v_{22}$ states are quite close together, there are probably strong interactions between them that would mix the states. This could explain the observation that the $R$-branch transitions are much weaker than the $P$-branch lines so that no transitions involving upper state $k l>0$ levels have been found. In addition there are quite a few other nearby binary vibrational states that could be involved, such as $v_{11}+v_{22}(E)$ near $2064 \mathrm{~cm}^{-1}, 2 v_{4}\left(A_{1}\right)$ near 2067 $\mathrm{cm}^{-1}, v_{3}+v_{4}\left(A_{1}\right)$ near $2071 \mathrm{~cm}^{-1}, 2 v_{3}\left(A_{1}\right)$ near $2074 \mathrm{~cm}^{-1}, 2 v_{22}\left(A_{1}, B_{1}, B_{2}\right)$ near $2116 \mathrm{~cm}^{-1}$ and $v_{5}+v_{21}(E)$ near $2120 \mathrm{~cm}^{-1}$. In view of this, speculation on the causes of the missing $\Delta k l=+1$ transitions seems unwarranted at this time. The constants resulting from the least-squares fit for this band are given in Table 2 .

\subsection{Other fundamental bands observed, but for which the rotational analysis was not attempted} or was unsuccessful.

No bands in the $\mathrm{CH}$ stretching region were examined since experience has shown these to be usually complex because of mixing with overtone and combination bands that can occur in this region. The spectra should be simpler at the low frequency extreme, where two fundamentals are predicted, $v_{12}\left(B_{1}\right)$ at $292 \mathrm{~cm}^{-1}$ and a perpendicular band, $v_{23}(E)$ at $308 \mathrm{~cm}^{-1}$. Some weak structure was seen near $300 \mathrm{~cm}^{-1}$ but we were unsuccessful in assigning it, possibly due to overlapping hot-band contributions and relatively poor signal-to-noise in the spectra.

A parallel band, $v_{17}$, and a perpendicular band, $v_{23}$, are expected to occur near $880 \mathrm{~cm}^{-1}$ and the spectrum does show very strong absorption in this region, with a $Q$-branch at about 871 $\mathrm{cm}^{-1}$. Unfortunately, most of the high resolution spectrum of this region is very cluttered and does not show the type of order that one would expect for a parallel band, nor does it show a pattern that can be identified with assignable transitions for a perpendicular band. Only in a region at about $868 \mathrm{~cm}^{-1}$ does a pattern emerge that looks like a $P$-branch for a parallel band, presumably $v_{17}$. However, no corresponding $R$-branch transitions could be identified, although there are many transitions in the $R$-branch region (but no apparent order). Since the $v_{23}$ fundamental is expected to be very close to $870 \mathrm{~cm}^{-1}$ one might expect to observe a strong $\Delta k=$ $\pm 1, \Delta l= \pm 1$ Coriolis interaction, but efforts to include this in the analysis were not enlightening. We note that two other states are predicted by the Gaussian calculations to occur in the $880 \mathrm{~cm}^{-1}$ 
region, $v_{5}+v_{12}\left(B_{1}\right)$ at $889 \mathrm{~cm}^{-1}$ and $v_{5}+v_{25}(E)$ at $896 \mathrm{~cm}^{-1}$. Both of these can interact with both $v_{17}$ and $v_{23}$, with dramatic effects on the intensity patterns, so the complexity of the bands is perhaps not surprising.

A similar difficulty occurs for the perpendicular $v_{21}$ band, which appears as a weak feature at about $1150 \mathrm{~cm}^{-1}$ In addition to $v_{21}$, predicted at $1158 \mathrm{~cm}^{-1}$, other states predicted in this region are $v_{8}+v_{12}\left(B_{2}\right), v_{8}+v_{25}(E), v_{12}+v_{17}\left(A_{2}\right)$ and $v_{12}+v_{23}(E)$ at 1132, 1148, 1164 and 1173 $\mathrm{cm}^{-1}$, respectively. The spectrum shows a complex mix of lines with no discernable pattern that could aid in making a rotational analysis.

Finally, according to our Gaussian predictions, there should be three fundamental vibrations near $1400 \mathrm{~cm}^{-1}, v_{2}\left(A_{1}\right), v_{15}\left(B_{2}\right)$ and $v_{20}(E)$, predicted at 1457,1402 and $1431 \mathrm{~cm}^{-1}$, respectively. The latter two are both Raman and infrared active and are consistent with the observation in the liquid of strong Raman bands near 1397 and $1427 \mathrm{~cm}^{-1}$. In our infrared spectra we see no sign of a parallel band in this region but we can fit some transitions to a perpendicular pattern for a band which is presumed to be $v_{20}$, with a ${ }^{r} Q_{0}$ sub-band observed at $1428.15 \mathrm{~cm}^{-1}$. Judging by the quickly-changing shape of the $Q$-branches, the upper-state for this band must be perturbed by some other nearby state possibly $v_{5}+v_{8}\left(A_{2}\right)$ or $v_{7}+v_{12}\left(B_{2}\right)$, predicted at 1426 and $1436 \mathrm{~cm}^{-1}$, respectively. Both could Coriolis-couple with $v_{20}$, making a detailed rotational analysis problematic.

\section{Discussion}

\subsection{Comparison of the observed constants with those given by the Gaussian calculation}

Table 1 lists the experimental values for the ground state constants and it is considered remarkable that just four parameters suffice to fit more than 5600 level spacings to a standard

deviation of only $0.00037 \mathrm{~cm}^{-1}$. (Many of these are of course duplicate combination-differences due to the use of data from several bands.) It is seen that $B_{0}$ is within $0.5 \%$ of the theoretical prediction and the two quartic centrifugal constants $D_{J}$ and $D_{J K}$ differ from theory by only 1.7 and $0.8 \%$, respectively. This agreement justifies fixing $A_{0}$ and $D_{K}$ constants at the theoretical values in the fitting process since, although the transition frequencies are independent of $A_{0}$ and $D_{K}$, the calculated line intensities are not.

Good agreement is also seen for the small $K=2$ splitting constant $\Delta_{2}$, which for the ground state can be taken to be very close to the value of $d_{2}$ computed for the equilibrium 
structure. The quartic distortion constants, including $d_{2}$, depend only on the harmonic potential energy terms and, in the $D_{2 d}$ case of spiropentane, theory [16] predicts that $d_{2}=\left(\tau_{\mathrm{xxxx}}+\tau_{\mathrm{yyyy}}-\right.$ $\left.2 \tau_{\mathrm{xxyy}}\right) / 64$, where the Wilson-Howard [17] $\tau$ constants depend only on the derivatives of the moments of inertia with respect to the normal modes. The Gaussian values are $\tau_{\mathrm{xxxx}}=\tau_{\mathrm{yyyy}}=$ -

$0.871 \times 10^{-7} \mathrm{~cm}^{-1}$ and $\tau_{\text {xxyy }}=-1.260 \times 10^{-7} \mathrm{~cm}^{-1}$, giving a positive value of $+1.22 \times 10^{-9}$ for $d_{2}$. This is within $4.1 \%$ of the experimental value which, with our level-ordering convention, is also of positive sign.

For excited vibrational states, theoretical calculations at the anharmonic level yield improved estimates of the vibrational frequencies $v$, in most cases within $1 \%$. However larger deviations can occur when Fermi and Coriolis interactions occur between nearby states and more recent versions of the Gaussian programs attempt to account for these. This accounts for some of the variability found in Gaussian anharmonic frequencies when using different versions of the Gaussian program.

Theory also leads to predictions of the changes in the rotational constants $\Delta A=A^{\prime}-A^{\prime \prime}=$ $-\alpha_{A}$ and $\Delta B=B^{\prime}-B^{\prime \prime}=-\alpha_{B}$ with vibrational state. The latter constants depend only on the quadratic and cubic terms in the potential and, for a given state, generally have the correct sign and are within about $20 \%$ of the experimental values. Such is the case for $v_{24}$, where agreement is better than $10 \%$ because there are no interactions with nearby states. However poorer agreement occurs for most of the other cases in Table 5 and the agreement of $A \zeta$ values is also generally poorer than normal, due to such interactions.

\subsection{The observed splitting constants}

As shown in Figure 3, the degenerate states of a molecule with $D_{2 d}$ symmetry such as spiropentane have both $k l=-1$ and $k l=+1$ levels split by terms that we have labeled $q^{-}$and $q^{+}$. Those same constants also result in a smaller, but observable splitting of the $k l=-3$ and $k l=+3$ levels. In the case of allene the $A$ value is so large that the coupling between the $K=1$ and $K=3$ levels is too small to cause a measureable splitting of the $K=3$ levels. For allene the analysis used intensity arguments to determine which levels are $A_{1}$ or $A_{2}$ or $B_{1}$ or $B_{2}$ but for spiropentane the intensity difference is smaller, $76 / 60$ vs $2 / 1$, and there are very many unidentified hot-band and overlapping transitions so the intensity measurements are less reliable.

As mentioned above, the infrared tovibrational selection rules allow $A_{1} \leftrightarrow B_{1}, A_{2} \leftrightarrow B_{2}$, and 
$E \leftrightarrow E$ transitions only and, as can be seen in Figure 3, the ${ }^{r} Q_{0}$ transitions will all involve upper state levels that are different from the levels that are reached by the ${ }^{r} R_{0}$ and ${ }^{r} P_{0}$ transitions. That provides a method of determining the order of the split $B_{1}$ and $B_{2}$ levels for both $k l=+1$ and $k l=$ -3 without making any intensity measurements. Intensity measurements are needed for the $A_{1} / A_{2}$ splitting and this proved possible for many lines of the $v_{24}$ band. Consequently we are confident that Figure 3 gives the correct ordering of the $A_{1}$ and $A_{2}$ levels as well as the $B_{1}$ and $B_{2}$ levels, both for the upper state, $v_{24}$, and for the ground state. The other perpendicular band that was studied, $v_{22}$, was found to have the order of the $k l=+1 B_{1}$ and $B_{2}$ levels reversed compared to $v_{24}$, as was the case for the $k l=-1 A_{1}$ and $A_{2}$ levels. The experimental results (in $\mathrm{cm}^{-1}$ ) are $v_{22}$ : $q^{+}=0.000114, q^{-}=-0.000012 ; \quad v_{24}: q^{+}=-0.000138, q^{-}=0.000549$.

The $q$ constants and the $\alpha$ 's are of comparable magnitude and both depend upon quadratic and cubic potential terms, with no dependence on quartic terms. Comparisons of experimental $q^{+}$and $q^{-}$values with theoretical Gaussian values would be desirable but these values are not computed in the program. In principle one can obtain the signs and magnitudes of these using the theoretical relations of Grenier-Besson [18] and the cubic force constants and other parameters generated by Gaussian. In practice we have found this difficult because of uncertainty regarding the labeling of the symmetries of the components of degenerate vibrations; efforts to resolve this and some other inconsistencies in the Gaussian output are underway.

\section{Acknowledgments}

J. Nibler acknowledges a Camille and Henry Dreyfus Senior Scientist Mentor Award which provided support of undergraduates Joseph Price and Jared Harzan. The infrared spectra were recorded at the Environmental Molecular Sciences Laboratory, a national scientific user facility sponsored by the Department of Energy's Office of Biological and Environmental Research and located at Pacific Northwest National Laboratory (PNNL). PNNL is operated for the United States Department of Energy by the Battelle Memorial Institute under contract DEAC05-76RLO 1830. We thank Robert Sams of PNNL for helpful advice and assistance in recording the infrared spectra of spiropentane at this facility. 


\section{References}

1. S. W. Benson, Thermochemical Kinetics, 2nd. ed., (1976) p. 273, Wiley, New York.

2. M.J. Murray, E.H. Stevenson, J. Am. Chem. Soc. 66 (1944) 812-816.

3. F.F. Cleveland, M.J. Murray, W.S. Gallaway, J. Chem. Phys. 15 (1947) 742-758.

4. G.R. Burns and D.G. McGavin, Appl. Spectrosc. 26 (1972) 540-542.

5. L.J. Schaad and A.A. Jarzęcki, J. Mol. Struct. (Theochem) 341 (1995) 271-278.

6. J.E. Price, K.A. Coulterpark, T. Masiello, J.W. Nibler, A. Weber, A. Maki, T.A. Blake, J. Mol. Spec. 269 (2011) 129-136.

7. L.S. Rothman et al. HITRAN and J. Quant. Spectrosc. Radiat. Transfer 96 (2005) 139-204.

8. A.G.Maki, J.S. Wells, Wavenumber Calibration Tables from Heterodyne Frequency Measurements, NIST Specieal Publication 821, US Department of Commerce, 1991. <http://physics.nist.gov/PhysRefData/wavenum/html/contents.html> (accessed 1995).

9. G.J. Cartwright and I.M. Mills; J. Mol. Spec., 34 (1970) 415-439.

10. F. Hegelund, N. Andresen and M. Koivusaari. J. Mol. Spec., 149 (1991) 305-313; 159 (1993) 230-248.

11. S. Nissen, F. Hegelund, M. S. Johnson, and B. Nelander, J. Mol. Spec., 216 (2002) 51-61.

12. J.W. Nibler, A. Weber, and A. Maki, A. Weber, J.W. Nibler, T. Masiello, T.A. Blake, R. Kirkpatrick, J. Mol. Spec. 264 (2010) 26-36

13. R. Kirkpatrick, T. Masiello, M. Martin, J.W. Nibler, A. Maki, A.Weber, and T.A. Blake, J. Mol. Spec. 281 (2012) 51-62.

14. B.N, Taylor, C.E. Kuyatt, NIST Technical Note No. 1297 (1994), Guidelines for Evaluating and Expressing the Uncertainty of NIST Measurement Results. This publication may be downloaded from http://physics.nist.gov/Pubs/guidelines/contents.html.

15. A. Perry, M.A. Martin, J.W. Nibler, A.Maki, A. Weber, and T.A. Blake, J. Mol. Spectrosc. 22-23 (2012) 22-32.

16. J. K. G. Watson, in Vibrational Spectra and Structure, Vol. 6, J. Durig, Ed., Elsevier, Amsterdam, (1997) 1-24.

17. E.B. Wilson Jr. and J.B. Howard. J. Chem. Phys., 4 (1936) p. 260. 
18. M.L. Grenier-Besson, J. Phys. Radium, 21 (1960) 555-565. 


\section{LIST OF TABLES}

Table 1 Rotational parameters (in $\mathrm{cm}^{-1}$ ) for the ground state of spiropentane.

Table 2. Rovibrational parameters $\left(\right.$ in $\mathrm{cm}^{-1}$ ) for $v_{22}, v_{24}$, and $v_{4}+v_{22}$ of spiropentane.

Table 3 Rovibrational parameters (in $\mathrm{cm}^{-1}$ ) for $v_{16}$ and $2 v_{22}$ of spiropentane.

Table 4. Rovibrational parameters (in $\mathrm{cm}^{-1}$ ) for $v_{14}$ and for either $2 v_{24}$ or $v_{5}+v_{16}$ of spiropentane.

Table 5. Comparison of some experimental and theoretical rovibrational parameters for spiropentane.

\section{LIST OF FIGURES}

Figure 1. The observed and calculated spectrum of the $v_{24}$ perpendicular fundamental band of spiropentane. The assigned $P, Q$, and $R$ line sequences are shown with dots in the lower trace.

Figure 2. An enlarged view of a segment of $v_{24}$ showing some of the ${ }^{r} Q_{2}$ transitions of spiropentane that are split into $A_{1} \leftarrow B_{1}$ and $A_{2} \leftarrow B_{2}$ transitions with respective relative intensities 76 to 60 as predicted from the nuclear spin weights. Also indicated are ${ }^{r} Q_{3}$ and ${ }^{r} Q_{4}$ sequences that show no splittings.

Figure 3. Energy level diagram for the $v_{24}(E)$ state and for the ground state $\left(A_{1}\right)$ of spiropentane. The order of the levels, i.e. which levels are above the other levels, is correct, but the scale is not. The number in parentheses gives the nuclear spin weight of the level. Allowed infrared rovibrational transitions are $A_{1} \leftarrow B_{1}, A_{2} \leftarrow B_{2}$ and $E \leftarrow E$; a few $\Delta J=0, \Delta K= \pm 1 Q$-branch transitions are shown.

Figure 4. The observed and calculated parallel bands of spiropentane in the 1550 $\mathrm{cm}^{-1}$ region. For display purposes, a number of intense water vapor lines have been deleted in the spectrum. The lower wavenumber band is $v_{14}$. The calculated upper band is for $v_{5}+v_{16}$, but a nearly identical simulation is obtained for $2 v_{24}\left(B_{2}\right)$, whose assignment is slightly favored (see text). 
$v_{24}(e)$ perpendicular band
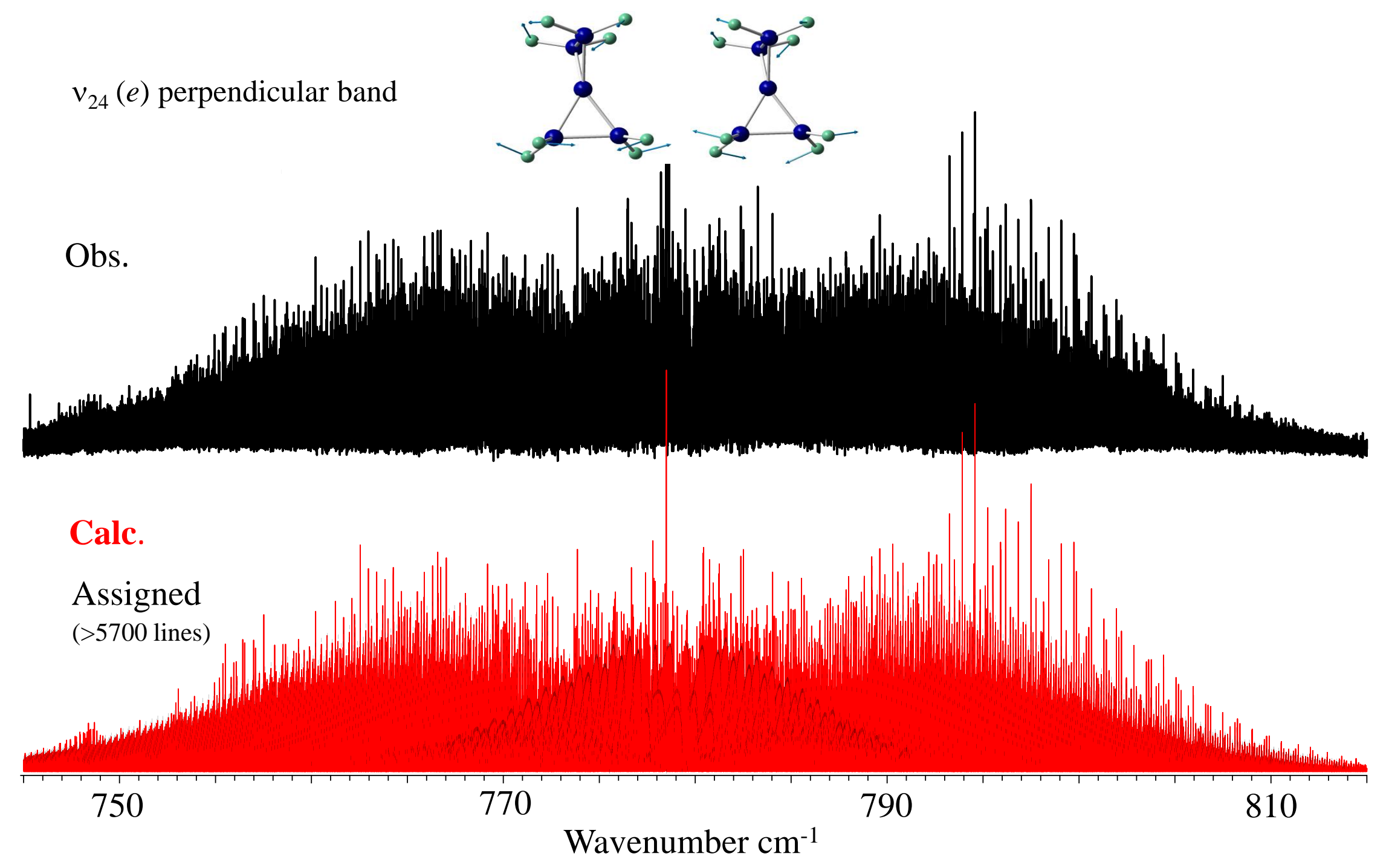


$$
v_{24}-{ }^{r} Q_{K^{\prime \prime}}\left(J^{\prime \prime}\right) \text { lines }
$$

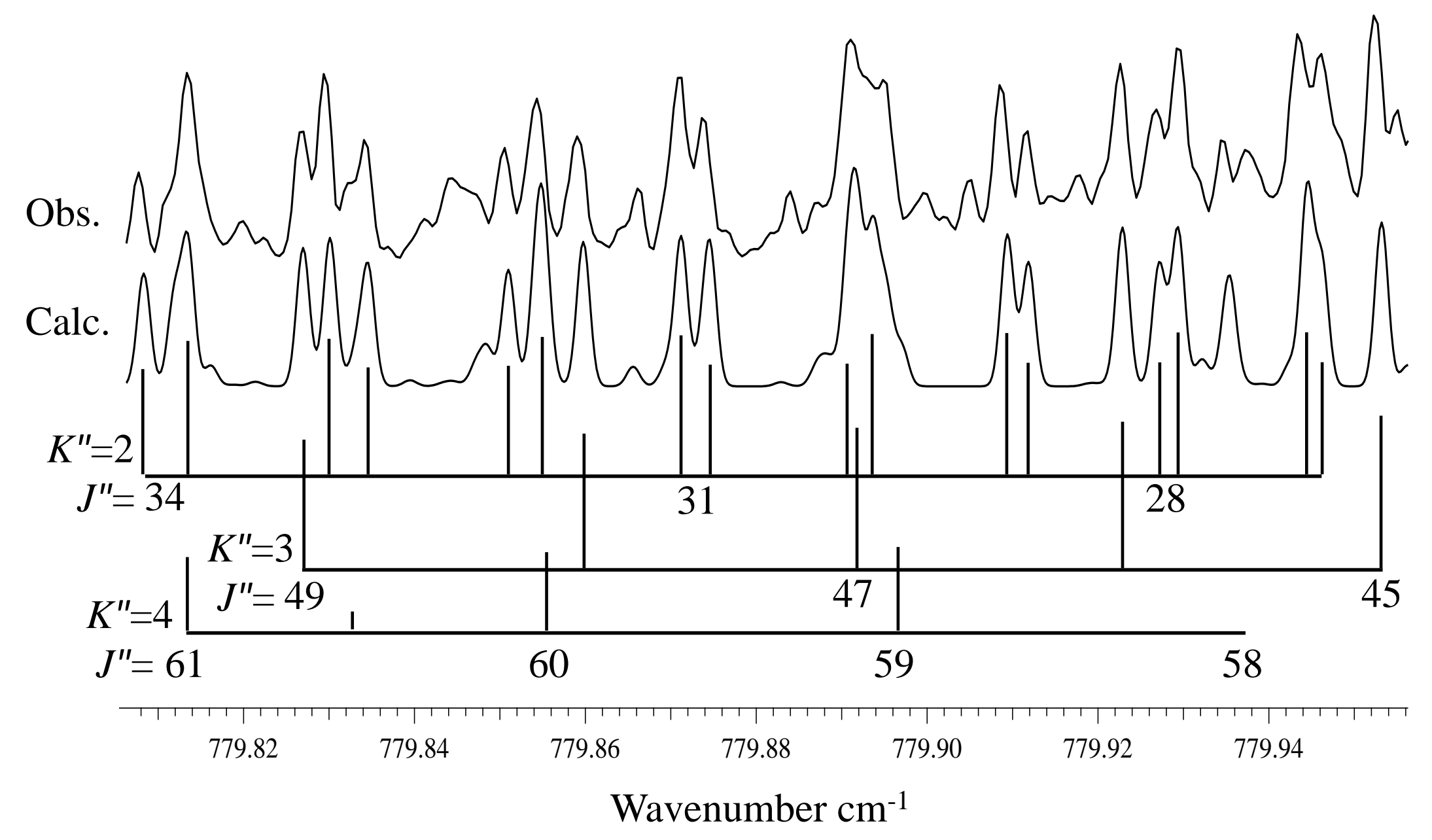




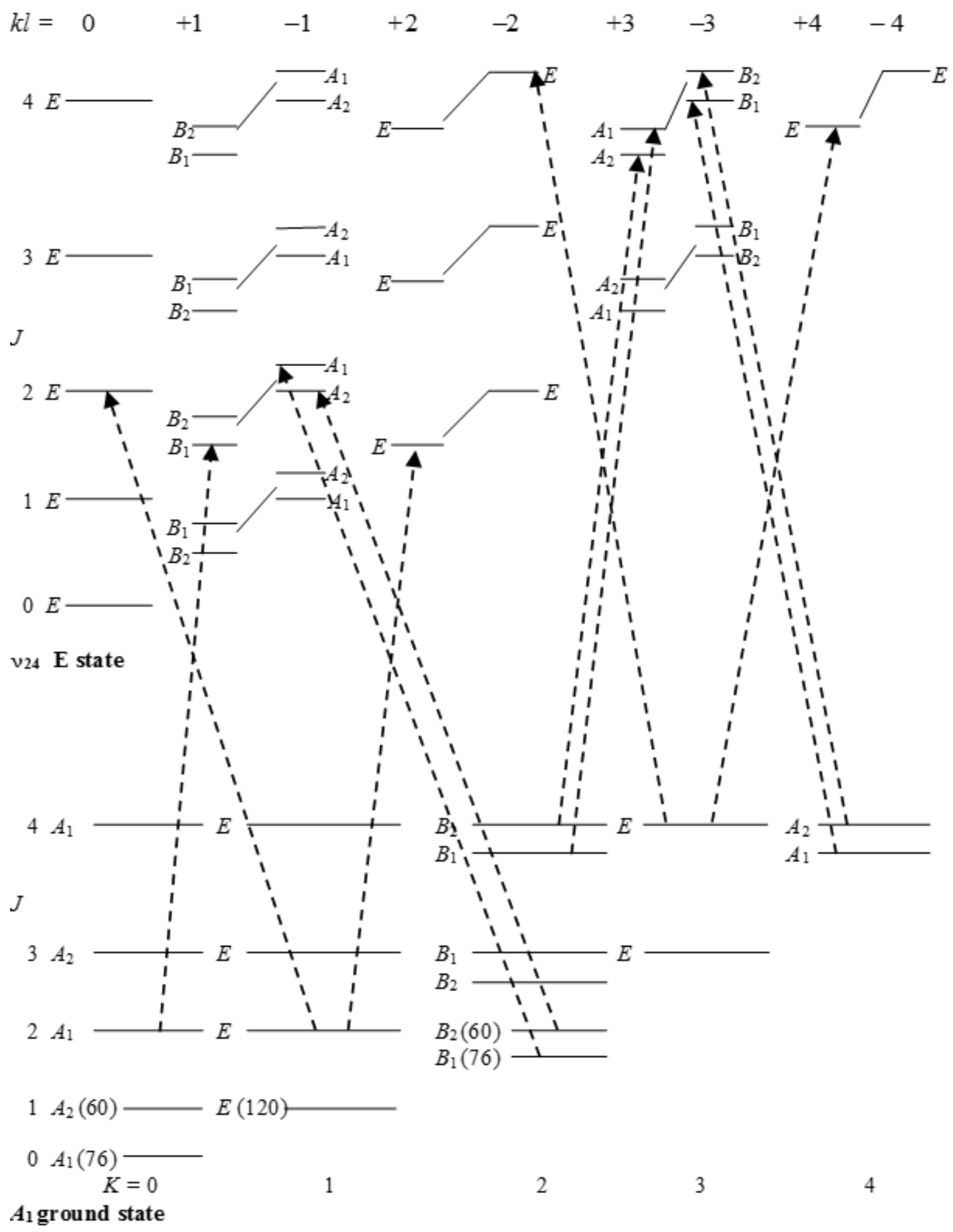


Calc.
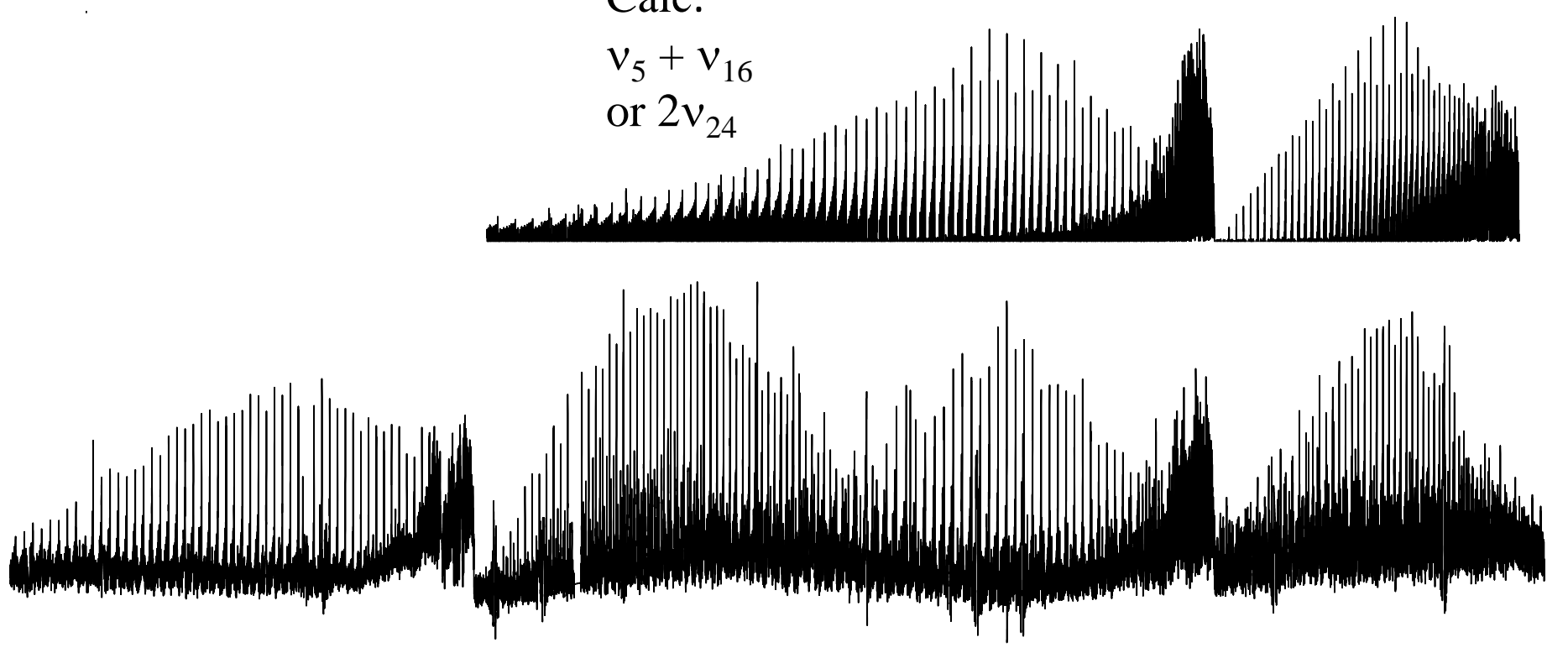

Calc. $v_{14}$

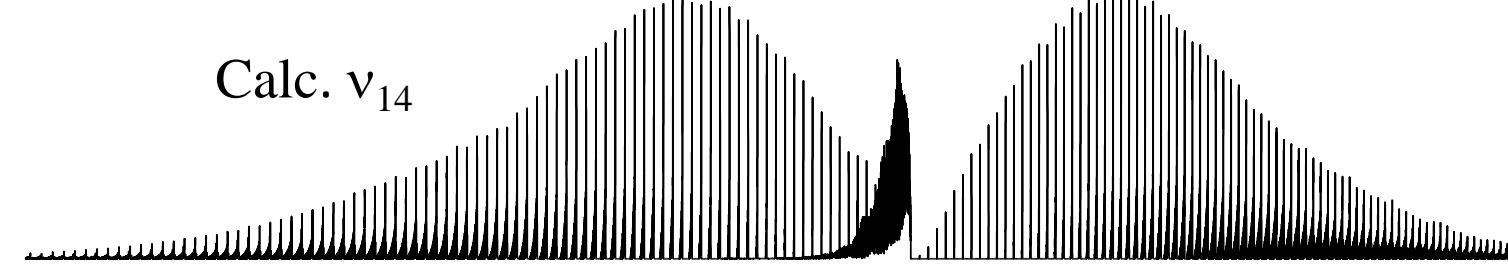

$\begin{array}{ccccccc}1520 & 1530 & 1540 & 1550 & 1560 & 1570 & 1580 \\ & \text { Wavenumber cm } & \end{array}$


Table 1

Rotational parameters (in $\mathrm{cm}^{-1}$ ) for the ground state of spiropentane

\begin{tabular}{lcc}
\hline Parameter $^{a}$ & Experimental & $\begin{array}{c}\text { Theoretical } \\
\text { B3LYP/cc-pVTZ }\end{array}$ \\
\hline$A_{0}$ & {$[0.35059]$} & 0.35059 \\
$B_{0}$ & $0.13947411(15)$ & 0.13877 \\
$D_{J} \times 10^{8}$ & $2.4615(15)$ & 2.420 \\
$D_{J K} \times 10^{8}$ & $8.66(4)$ & 8.590 \\
$D_{K} \times 10^{8}$ & {$[5.21]^{b}$} & 5.21 \\
$H_{J} \times 10^{13}$ & {$[0.0]$} & 0.053 \\
$H_{J K} \times 10^{13}$ & {$[0.0]$} & -5.45 \\
$H_{K J} \times 10^{13}$ & {$[0.0]$} & 8.99 \\
$H_{K} \times 10^{13}$ & {$[0.0]$} & -3.90 \\
$\Delta_{2} \times 10^{9}$ & $1.172(16)$ & 1.22 \\
& & \\
$J_{\max }=$ & 96 & \\
$K_{\max }=$ & 31 & \\
$\#$ differences & 5612 & \\
rms dev. $\left(\mathrm{cm}^{-1}\right)$ & 0.00037 & \\
\hline
\end{tabular}

${ }^{a}$ Given in parentheses here and in subsequent tables are uncertainties in the last digits (twice the standard deviation = type B, $\mathrm{k}=2$, uncertainties as defined in Ref. [14].)

${ }^{b}$ Values given in square brackets were held fixed. 
Table 2

Rovibrational parameters (in $\mathrm{cm}^{-1}$ ) for $v_{22}, v_{24}$, and $v_{4}+v_{22}$ of spiropentane

\begin{tabular}{|c|c|c|c|}
\hline Parameter $^{a}$ & $v_{22}$ & $v_{24}$ & $v_{4}+v_{22}$ \\
\hline$v_{0}$ & $1051.36066(4)$ & $779.12623(3)$ & $2081.52595(19)$ \\
\hline$\Delta A \times 10^{3}$ & $-0.2534(4)$ & $-0.65283(12)$ & $-0.699(9)$ \\
\hline$\Delta B \times 10^{3}$ & $-0.08790(6)$ & $-0.28430(6)$ & $-0.14297(24)$ \\
\hline$\Delta D_{J} \times 10^{8}$ & $0.3662(22)$ & $-0.2439(33)$ & $0.247(7)$ \\
\hline$\Delta D_{J K} \times 10^{8}$ & $-2.792(16)$ & $2.300(10)$ & $3.15(32)$ \\
\hline$\Delta D_{K} \times 10^{8}$ & $1.28(8)$ & $-2.038(12)$ & $31.1(9)$ \\
\hline$\Delta H_{J} \times 10^{13}$ & {$[0.0]^{b}$} & $-0.454(58)$ & {$[0.0]$} \\
\hline$\Delta H_{J K} \times 10^{13}$ & {$[0.0]$} & $9.92(25)$ & {$[0.0]$} \\
\hline$\Delta H_{K J} \times 10^{13}$ & {$[0.0]$} & $-26.2(5)$ & $-102 .(26)$ \\
\hline$\Delta H_{K} \times 10^{13}$ & {$[0.0]$} & $17.8(4)$ & {$[0.0]$} \\
\hline$A \zeta$ & $-0.0017402(26)$ & $0.0022338(10)$ & $-0.00179(4)$ \\
\hline$\eta_{J} \times 10^{5}$ & $0.1129(6)$ & $0.21260(24)$ & $0.0141(52)$ \\
\hline$\eta_{K} \times 10^{5}$ & $-0.1329(18)$ & $-0.21500(29)$ & $-1.29(5)$ \\
\hline$\eta_{J J} \times 10^{10}$ & $-0.085(21)$ & $0.616(11)$ & {$[0.0]$} \\
\hline$\eta_{J K} \times 10^{10}$ & $2.49(13)$ & {$[0.0]^{c}$} & {$[0.0]$} \\
\hline$\eta_{K} \times 10^{10}$ & {$[0.0]$} & $-0.564(13)$ & {$[0.0]$} \\
\hline$q^{+} \times 10^{3}$ & $0.1138(5)$ & $-0.13780(19)$ & {$[0.0]$} \\
\hline$q^{+}{ }_{J} \times 10^{8}$ & $0.559(24)$ & $0.093(8)$ & {$[0.0]$} \\
\hline$q^{+}{ }_{K} \times 10^{8}$ & {$[0.0]$} & $-17.87(19)$ & {$[0.0]$} \\
\hline$q^{-} \times 10^{3}$ & $-0.0124(4)$ & $0.54922(12)$ & $-0.0137(4)$ \\
\hline$q^{-} \times 10^{8}$ & $-2.403(24)$ & $-0.456(5)$ & {$[0.0]$} \\
\hline$q^{-}{ }_{K} \times 10^{8}$ & {$[0.0]$} & $19.81(11)$ & {$[0.0]$} \\
\hline$w_{4,0} \times 10^{8}$ & {$[0.0]$} & $-0.1366(16)$ & {$[0.0]$} \\
\hline$J_{\max }=$ & 69 & 74 & 55 \\
\hline$K_{\max }=$ & 24 & 52 & 31 \\
\hline \# transitions & 3370 & 5709 & 1143 \\
\hline $\mathrm{rms} \operatorname{dev} .\left(\mathrm{cm}^{-1}\right)$ & 0.00039 & 0.00027 & 0.00054 \\
\hline
\end{tabular}

${ }^{a} \Delta B=B^{\prime}-B^{\prime \prime}, \Delta A=A^{\prime}-A^{\prime \prime}$, etc.

${ }^{b}$ Values given in square brackets were held fixed. 
Table 3

Rovibrational parameters ${ }^{a}\left(\right.$ in cm $^{-1}$ ) for $v_{16}$ and $2 v_{22}$ of spiropentane

\begin{tabular}{lll}
\hline Parameter & \multicolumn{1}{c}{$v_{16}\left(B_{2}\right)$} & \multicolumn{1}{c}{$2 v_{22}\left(B_{2}\right)$} \\
\hline$v_{0}$ & $992.53792(3)$ & $2098.21151(15)$ \\
$\Delta A \times 10^{3}$ & $-1.01921(21)$ & $-0.5860(26)$ \\
$\Delta B \times 10^{3}$ & $0.024718(25)$ & $-0.14225(20)$ \\
$\Delta D_{J} \times 10^{8}$ & $0.0335(6)$ & $0.596(5)$ \\
$\Delta D_{J K} \times 10^{8}$ & $0.436(8)$ & $-9.78(23)$ \\
$\Delta D_{K} \times 10^{8}$ & $-11.73(4)$ & $-14.22(30)$ \\
$\Delta H_{J} \times 10^{13}$ & $0.022(5)$ & {$[0.0]^{b}$} \\
$\Delta H_{J K} \times 10^{13}$ & $-0.75(7)$ & {$[0.0]$} \\
$\Delta H_{K J} \times 10^{13}$ & $20.8(6)$ & {$[0.0]$} \\
$\Delta H_{K} \times 10^{13}$ & $-141.1(18)$ & {$[0.0]$} \\
$\Delta_{2} \times 10^{9}$ & $-1.108(3)$ & {$[0.0]$} \\
$A \zeta$ & & $0.000001(10)$ \\
& & \\
$J_{\max }=$ & 103 & 68 \\
$K_{\max }=$ & 43 & 29 \\
$\#$ transitions & 3371 & 452 \\
rms dev. $\left(\mathrm{cm}^{-1}\right)$ & 0.00023 & 0.00042 \\
\hline \hline
\end{tabular}

${ }^{a} \Delta B=B^{\prime}-B^{\prime \prime}, \Delta A=A^{\prime}-A^{\prime \prime}$, etc.

${ }^{b}$ Values given in square brackets were held fixed. 


\section{Table 4}

Rovibrational parameters (in $\mathrm{cm}^{-1}$ ) for $v_{14}$ and for either $2 v_{24}$ or $v_{5}+v_{16}$ of spiropentane

\begin{tabular}{|c|c|c|c|c|}
\hline Parameter $^{a}$ & $v_{14}\left(B_{2}\right)$ & $2 v_{24}\left(B_{2}\right)$ & $2 v_{24}\left(A_{l}\right)$ & $v_{5}+v_{16}\left(B_{2}\right)$ \\
\hline$v_{0}$ & $1539.53206(14)$ & $1567.5223(3)$ & $1569.69(13)$ & $1567.5221(3)$ \\
\hline$\Delta A \times 10^{3}$ & $-0.6110(9)$ & $-1.190(9)$ & {$[-1.190]$} & $-1.394(6)$ \\
\hline$\Delta B \times 10^{3}$ & $-0.45443(12)$ & $-0.8520(13)$ & {$[-0.8520]$} & $-0.8500(12)$ \\
\hline$\Delta D_{J} \times 10^{8}$ & {$[0.0]^{b}$} & $8.84(14)$ & {$[8.84]$} & $9.02(14)$ \\
\hline$\Delta D_{J K} \times 10^{8}$ & $0.56(4)$ & $-3.2(12)$ & {$[-3.2]$} & $-37.4(12)$ \\
\hline$\Delta D_{K} \times 10^{8}$ & $0.62(17)$ & {$[0.0]$} & {$[0.0]$} & $-17.5(37)$ \\
\hline$\Delta H_{J} \times 10^{13}$ & {$[0.0]$} & $-187 .(5)$ & {$[-187]$.} & $-184 .(5)$ \\
\hline$\Delta H_{J K} \times 10^{13}$ & {$[0.0]$} & {$[0.0]$} & {$[0.0]$} & $-1240 .(51)$ \\
\hline$\Delta H_{K J} \times 10^{13}$ & {$[0.0]$} & {$[0.0]$} & {$[0.0]$} & $-3372 .(308)$ \\
\hline$\Delta H_{K} \times 10^{13}$ & $72 .(9)$ & {$[0.0]$} & {$[0.0]$} & 3069.(752) \\
\hline$A \zeta$ & & $0.000961(26)$ & & \\
\hline$\eta_{J} \times 10^{5}$ & & $0.210(10)$ & & \\
\hline$\eta_{J J} \times 10^{9}$ & & $-0.901(39)$ & & \\
\hline$q+\times 10^{4}$ & & $\pm 0.78(7)$ & & \\
\hline$J_{\max }=$ & 56 & 48 & & 48 \\
\hline$K_{\max }=$ & 40 & 17 & & 17 \\
\hline \# transitions & 453 & 416 & & 416 \\
\hline rms dev. $\left(\mathrm{cm}^{-1}\right)$ & 0.00039 & 0.00050 & & 0.00055 \\
\hline
\end{tabular}

${ }^{a} \Delta B=B^{\prime}-B^{\prime \prime}, \Delta A=A^{\prime}-A^{\prime \prime}$, etc.

${ }^{b}$ Values given in square brackets were held fixed. 
Table 5

Comparison of some experimental and theoretical rovibrational parameters $\left(\mathrm{cm}^{-1}\right)$ for spiropentane

\begin{tabular}{|c|c|c|c|c|c|c|c|c|c|c|}
\hline \multirow[t]{2}{*}{ Parameter $^{a}$} & \multirow[t]{2}{*}{ Symmetry } & \multicolumn{2}{|c|}{$v_{0}$} & \multirow{2}{*}{$\begin{array}{c}\text { Anharmonic } \\
\text { Intensity, } \\
\text { Theory }\end{array}$} & \multicolumn{2}{|c|}{$\Delta A \times 10^{3}$} & \multicolumn{2}{|c|}{$\Delta B \times 10^{3}$} & \multicolumn{2}{|c|}{$A \zeta$} \\
\hline & & Expt. & Theory $^{b}$ & & Expt. & Theory & Expt. & Theory & Expt. & Theory \\
\hline$v_{14}$ & $B_{2}$ & 1540 & 1527 & 0.32 & -0.611 & -0.424 & -0.454 & -0.466 & & \\
\hline$v_{16}$ & $B_{2}$ & 993 & 998 & 15.27 & -1.019 & -1.330 & 0.025 & 0.014 & & \\
\hline$v_{22}$ & $E$ & 1051 & 1059 & 2.04 & -0.253 & -0.233 & -0.088 & -0.061 & -0.0017 & -0.0022 \\
\hline$v_{24}$ & $E$ & 779 & 784 & 1.43 & -0.653 & -0.622 & -0.284 & -0.259 & 0.0022 & 0.0029 \\
\hline $2 v_{22}$ & $\boldsymbol{B}_{2}$ & 2098 & 2113 & 1.22 & -0.586 & -0.466 & -0.142 & -0.122 & & -0.0022 \\
\hline $2 v_{24}$ or & $\boldsymbol{B}_{2}$ & 1568 & 1579 & 0.02 & -1.182 & -1.244 & $-\mathbf{0 . 8 5 3}$ & -0.518 & 0.0009 & 0.0029 \\
\hline$v_{5}+v_{16} ?$ & $B_{2}$ & 1568 & 1587 & 0.42 & -1.392 & -0.871 & -0.852 & -0.085 & & \\
\hline$v_{4}+v_{22}$ or & $E$ & 2082 & 2092 & 1.33 & -0.698 & -0.833 & -0.143 & -0.131 & -0.0018 & -0.0022 \\
\hline$v_{3}+v_{22} ?$ & $E$ & 2082 & 2095 & 0.12 & -0.698 & -1.879 & -0.143 & 0.0781 & -0.0018 & -0.0022 \\
\hline$v_{5}+v_{14}$ or & $\boldsymbol{B}_{2}$ & 2146 & 2120 & 0.10 & $<0$ & 0.345 & -0.90 & -0.565 & & \\
\hline$v_{7}+v_{11} ?$ & $B_{2}$ & 2146 & 2149 & 0.07 & $<0$. & 0.763 & -0.90 & 0.063 & & \\
\hline
\end{tabular}

${ }^{a} \Delta B=B^{\prime}-B^{\prime \prime}, \Delta A=A^{\prime}-A^{\prime \prime}$. For cases with alternative assignments, those in boldface are favored.

${ }^{b}$ The theoretical results (B3LYP/cc-pVTZ) were calculated using the Anharm and Vibrot options of Gaussian G03W.E.01 except for the anharmonic $v_{0}$ and intensity values, which were calculated using Gaussian 09W.D.01. Italicized $v_{0}$ values were corrected by Gaussian for Fermi resonances. 


\section{$v_{24}$ rotation-vibration band of spiropentane}

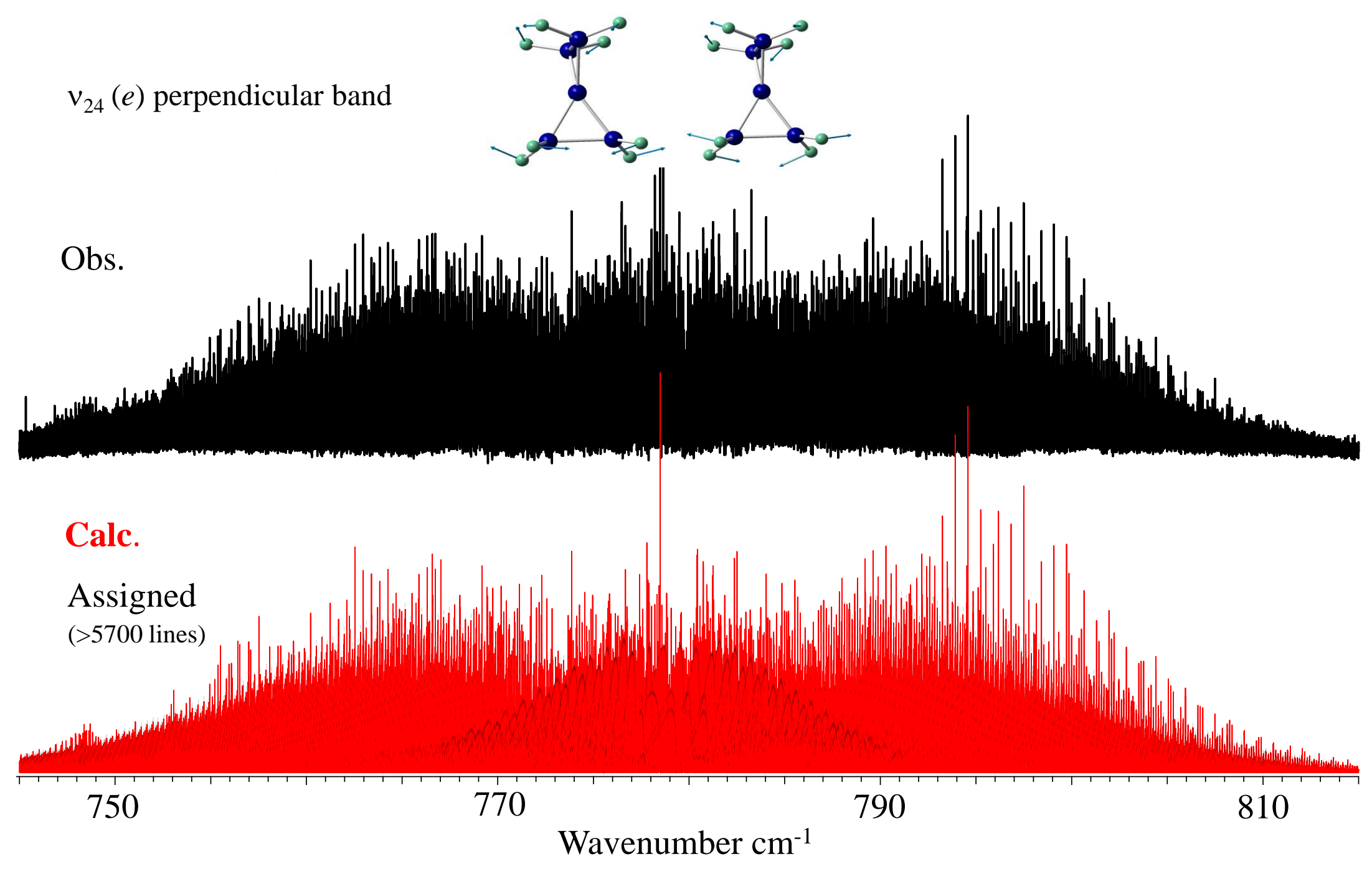

